\title{
ON THE FATOU SET OF MONIC POLYNOMIALS
}

\author{
P. BHATTACHARYYA and Y. E. ARUMARAJ
}

\section{Introduction}

The theory of iteration of a rational or entire function of a complex variable $z$ deals with the sequence of natural iterates $f_{n}(z)$ defined by

$$
f_{0}(z)=z, f_{n+1}(z)=f\left(f_{n}(z)\right), \quad n=0,1, \ldots
$$

In the theory developed by Fatou [3,4] and Julia [6], the central object of study is the Fatou Set $F(f)$ of those points of the complex plane in no neighbourhood of which the sequence of natural iterates $\left\{f_{n}(z)\right\}$ forms a normal family in the sense of Montel. Unless $f(z)$ is a rational function of order 0 or 1 , the set $F(f)$ has the following properties, proved for rational functions in [3] and for entire functions in [4].

I. $F(f)$ is a nonempty perfect set.

II. $F\left(f_{n}\right)=F(f)$ for any integer $n \geqq 1$.

III. $F(f)$ is completely invariant under the mapping $z \rightarrow f(z)$, i.e., if $\alpha$ belongs to $F(f)$, then $f(\alpha)$ and every solution $\beta$ of $f(\beta)=\alpha$ also belong to $F(f)$.

IV. $F(f)$ has no interior points unless it is the whole plane.

In the general theory a number of properties of the Fatou set have been derived [3, 4,6]. Myrberg [7] and Brolin [2] have studied the Fatou set of polynomials of degree 2 and 3 with real coefficients. References to Myrberg's other papers on the same subject can be found in [2]. Bhattacharyya and Arumaraj [1] have studied the Fatou sets for polynomials of degree 4 with real coefficients.

These authors have determined the structures of the Fatou sets for corresponding polynomials under suitable assumptions on the coefficients.

It is clear that if $f(z)=z^{k}, k \geqq 2$, then $F(f)$ is the unit circle. No example of a monic polynomial of the form $z^{k}+a_{1} z^{k-1}+\ldots+a_{k}, k \geqq 2$ has been found such that the corresponding Fatou set lies completely inside the unit disk. Thus two questions arise naturally:

1. Is it true that there exists a monic polynomial $f(z)$ of degree at least 2 such that

$$
\underset{z \in F(f)}{\operatorname{Max}}|z|<1 .
$$


2. Is it true that if $f$ is a monic polynomial and $F(f)=\{z:|z|=1\}$, then $f$ must be $z^{k}, k \geqq 2$ integer?

The aim of this paper is to answer these questions. We prove

Theorem 1. Let

$$
\begin{aligned}
& f(z)=z^{k}+a_{1} z^{k-1}+\ldots+a_{k}, \quad k \geqq 2 \\
& g(z)=z^{m}+b_{1} z^{m-1}+\ldots+a_{m}, \quad m \geqq 1
\end{aligned}
$$

be two monic polynomials. Then

$$
\operatorname{Max}_{z \in F(f)}|g(z)| \geqq 1 .
$$

Putting $g(z)=z$ in Theorem 1, we get

Corollary 1. Let $f(z)$ be a monic polynomial of degree at least 2 . Then there exists $z_{0} \in F(f)$ such that $\left|z_{\mathbf{0}}\right| \geqq 1$.

This shows that the answer to our first question is negative.

Theorem 2. Let $f(z)$ be a monic polynomial of degree at least 2. Let

$$
\operatorname{Max}_{z \in F(f)}|z|=1 \text {. }
$$

Then

$$
f(z)=z^{k}, \quad k \geqq 2 .
$$

This shows that the answer to our second question is positive.

Let $\sigma(F(f))$ and $\gamma(F(f))$ denote the Chebyshev's constant and the capacity of $F(f)$, where $f$ is a monic polynomial of degree at least 2. For definitions we refer to $[8]$, p. 71.

Fekete [8], p. 73 proves that $\sigma(F)=\gamma(F)$ for any set $F$. Brolin [2] has proved that $\gamma(F(f))=1$, where $F(f)$ is the Fatou set of a monic polynomial of degree at least 2 . Combining these two results, we immediately get

Theorem 3. The Chebyshev's constant $\sigma(F(f))=1$, where $F(f)$ is the Fatou set of a monic polynomial of degree at least 2.

We give an alternative proof of this theorem, which is elementary in the sense that we use only the notion of Chebyshev's constant and the property of the Fatou set for monic polynomials given by Theorem 2 .

We prove our theorems in Section 2 and discuss some consequences of Theorem 2 in Section 3. 


\section{Proof of the theorems}

First, we prove a lemma which is an easy consequence of the maximum modulus theorem.

Lemma 1. Let

and

$$
f(z)=z^{k}+a_{1} z^{k-1}+\ldots+a_{k}
$$

$$
g(z)=z^{m}+b_{1} z^{m-1}+\ldots+b_{m}
$$

be two monic polynomials of degree $k$ and $m$, respectively. Let $R>0$ and

Then

$$
E=\{z:|f(z)| \leqq R\} .
$$

Proof of Lemma 1.

$$
\operatorname{Max}_{z \in E}|g(z)| \geqq R^{m / k}
$$

We have

$$
\operatorname{Max}_{z \in E}|g(z)|^{k}=\max _{|f(z)|=R}|g(z)|^{k}=\max _{|f(z)|=R} R^{m} \frac{|g(z)|^{k}}{|f(z)|^{m}} .
$$

Now $h(z)=[g(z)]^{k} /[f(z)]^{m}$ is analytic in $\hat{C} \backslash E$, where $\hat{C}$ is the extended complex plane. Hence by maximum modulus principle

$$
\operatorname{Max}_{z \in E}|g(z)|^{k} \geqq R^{m} \lim _{z \rightarrow \infty}|h(z)|=R^{m},
$$

and the lemma follows on our taking the $k$-th root.

Proof of Theorem 1.

We can choose $R>1$ such that

$$
|f(z)|>R \text { for }|z|>R .
$$

Setting

and using (1) we get

$$
D_{-n}=\left\{z:\left|f_{n}(z)\right| \leqq R\right\}
$$

and

$$
D_{-(n+1)} \subset D_{-n}
$$

where

$$
F=F(f)=\partial D,
$$

$$
D=\bigcap_{n=0}^{\infty} D_{-n} .
$$

Since $R>1$, we have by Lemma 1 for every integer $n$,

$$
\operatorname{Max}_{z \in D_{-n}}|g(z)|=\operatorname{Max}_{\left|f_{n}(z)\right| \leqq R}|g(z)| \geqq R^{m / k^{n}} \geqq 1 .
$$


Hence

$$
\lim _{n \rightarrow \infty} \operatorname{Max}_{z \in D}|g(z)|=\operatorname{Max}_{z \in D}|g(z)|=\operatorname{Max}_{z \in \delta D}|g(z)|=\operatorname{Max}_{z \in F}|g(z)| \geqq 1,
$$

and the theorem is proved.

We next prove Theorem 3. We shall use the notion of Chebyshev's polynomial. For definition and the required results on Chebyshev's polynomial we refer to [8], pp. 71, 72 .

\section{Proof of Theorem 3.}

Let $T^{(n)}(z)$ be the Chebyshev's polynomial of $F(f)$ of degree $k^{n}$. Then $T^{(n)}(z)$ is a monic polynomial of degree $\geqq 2$. From Theorem 1 we get

$$
\operatorname{Max}_{z \in F(f)}\left|T^{(n)}(z)\right| \geqq 1 \text {. }
$$

It follows from the definition of Chebyshev's polynomial that

$$
\operatorname{Max}_{z \in F(f)}\left|T^{n}(z)\right| \leqq \operatorname{Max}_{z \in F(f)}\left|f_{n}(z)\right| .
$$

From (2) and (3) we get

$$
1 \leqq m_{k_{n}} \leqq R
$$

where

$$
m_{k^{n}}=\operatorname{Max}_{z \in F(f)}\left|T^{n}(z)\right|
$$

and

$$
R=\operatorname{Max}_{z \in F(f)}|z|=\operatorname{Max}_{z \in F(f)}\left|f_{n}(z)\right|, \quad n=1,2, \ldots
$$

It follows from (4) that

$$
\lim _{n \rightarrow \infty}\left(m_{k^{n}}\right)^{1 / k^{n}}=1
$$

i.e.,

$$
\lim _{n \rightarrow \infty} m_{n}^{1 / n}=1
$$

i.e.,

and the theorem is proved.

$$
\sigma(F)=1
$$

Proof of Theorem 2.

Let $g^{(n)}(z)$ be any monic polynomial of degree $k^{n}$. Then we have by Theorem 1

$$
\operatorname{Max}_{z \in F(f)}\left|g^{(n)}(z)\right| \geqq 1
$$

Also, by our assumption

$$
\underset{z \in F(f)}{\operatorname{Max}}\left|f_{n}(z)\right|=\operatorname{Max}_{z \in F(f)}|z|=1 .
$$

From (1) and (2) we get

$$
\operatorname{Max}_{z \in F(f)}\left|f_{n}(z)\right| \leqq \operatorname{Max}_{z \in F(f)}\left|g^{(n)}(z)\right| \text { for } n=0,1,2, \ldots
$$


If $T^{(n)}(z)$ denotes the Chebyshev's polynomial of $F(f)$ of degree $k^{n}$, then it follows from (6) that

$$
T^{(n)}(z)=f_{n}(z), \quad n=0,1,2, \ldots
$$

Since

(5) becomes

$$
\underset{z \in F(f)}{\operatorname{Max}}\left|z^{k^{n}}\right|=1 \quad \text { for every } n,
$$

$$
\operatorname{Max}_{z \in F(f)}\left|z^{k^{n}}\right| \leqq \operatorname{Max}_{z \in F(f)}\left|g^{(n)}(z)\right| \quad n=0,1,2, \ldots .
$$

We get from (9)

$$
T^{(n)}(z)=z^{k^{n}} .
$$

Since $T^{(n)}(z)$ is unique ([8], p. 72), it follows from (8) and (10) that

$$
T^{(n)}(z)=f_{n}(z)=z^{k^{n}}, \quad n=0,1,2, \ldots .
$$

Putting $n=1$ in (11), we get

and the theorem is proved.

$$
f(z)=f_{1}(z)=z^{k},
$$

\section{Some consequences}

If $E$ is closed and bounded and of transfinite diameter $d$, then the exterior Jordan content of $E$ is $\leqq \pi d^{2}$. This is an old result of Pólya (see e.g. Golusin [4], Chapter 7). Thus in the case where $F(f)$ is a Jordan curve the content of its interior is $\leqq \pi d^{2}$. Our Theorem 2 shows that the result of Pólya is sharp.

The exterior linear Jordan measure of the orthogonal projection of a closed set $E$ on any line is $\leqq 4 d$, where $d$ is the transfinite diameter of $E$ (see e.g. Golusin [5]).

Let now $E=F(f)$ be the Fatou set of a monic polynomial $f$ and let $E$ be connected. If $E$ has ordinary diameter $\mu$ and $z_{1}$ and $z_{2}$ are points of $E$ at a distance $\mu$, the projection of $E$ on the line joining $z_{1}$ and $z_{2}$ is exactly the segment $z_{1} z_{2}$ by the connectivity of $E$. Thus we get

$$
\mu \leqq 4 d .
$$

Now it is well known that for any set the transfinite diameter and the Chebyshev's constants are the same [8], p. 73. Hence by Theorem 3 we get $\mu \leqq 4$, i.e., we have proved:

Corollary 2. Let $f$ be a monic polynomial such that $F(f)$ is connected. Let $\mu(F)$ denote the diameter of $F(f)$. Then

$$
\mu(F) \leqq 4 .
$$

This is sharp as is shown by the example $f(z)=z^{2}-2$, for which $F(f)$ is the interval [-2, 2] (Myrberg [7], p. 12). 
The assumption that $F(f)$ is connected cannot be relaxed. For, if $f(z)=z^{2}-12$, then $F(f)$ is not connected (Brolin [2], Theorem 12.1, p. 126). Clearly $\pm 4 \in F(f)$ in this case.

From Theorem 2 it is clear that if $f$ is a monic polynomial not of the form $z^{k}$, then

$$
\mu(F(f))>1 \text {. }
$$

It is interesting to see what the best possible lower bound of $\mu F(f)$ is for such polynomials. We conjecture that if $f(z)=z^{n}+a_{1} z^{n-1}+\ldots+a_{n}$ is a monic polynomial of degree $\geqq 2$, then

$$
\mu(F(f)) \geqq 2 .
$$

We conclude our work making the following observations in support of our conjecture.

For a monic polynomial $f$ of degree $\geqq 2, F(f)$ is compact. Let $\mu$ be the exact diameter of $F(f)$. The compactness of $F(f)$ allows us to find two points $z_{1}$ and $z_{2}$ belonging to $F(f)$ such that $\left|z_{1}-z_{2}\right|=\mu$.

The line joining $z_{1}$ and $z_{2}$ contains no points of $F(f)$ outside the segment $z_{1} z_{2}$. Assume $\mu<2$ and take

$$
\alpha=\frac{z_{1}+z_{2}}{2}, g(z)=z-\alpha
$$

in Theorem 1. Then there exists a point $p$ of $F(f)$ such that

Then

$$
|p-\alpha| \geqq 1
$$

$$
\begin{aligned}
& \left|z_{1}-p\right| \leqq \mu \\
& \left|z_{2}-p\right| \leqq \mu .
\end{aligned}
$$

Without loss of generality, we assume that $p$ and $z_{1}$ lie on the same side of the perpendicular bisector of $z_{1}$ and $z_{2}$. Then

Then,

$$
\left|z_{2}-p\right|>\left(1+\left(\mu^{2} / 4\right)\right)^{1 / 2} .
$$

$$
\left(1+\left(\mu^{2} / 4\right)\right)^{1 / 2} \leqq \mu, \text { i.e., } \quad \mu>1.15 .
$$

I. N. Baker has pointed out that by more elaborate arguments one can get as much as $\mu>1.5$ and that one can perhaps go as far as $\mu \geqq r^{\prime}$. But one will not go beyond this by using the above method which assumes that any disc of radius 1 contains points of $F$ in its exterior.

The authors wish to thank Dr. I. N. Baker of Imperial College, London for his many valuable suggestions and comments during the preparation of this paper. 


\section{References}

[1] Bhattacharyya, P., and Y. E. Arumaraj: On the iteration of polynomials of degree 4 with real coefficients. - Ann. Acad. Sci. Fenn. Ser A I Math. 6, 1981, 197-203.

[2] Brolin, H.: Invariant sets under iteration of rational functions. - Ark. Mat. 6, 1965-67 $103-144$.

[3] Fatou, P.: Sur les équations fonctionnelles. - Bull. Soc. Math. France 47, 1919, 161-271, and $48,1920,33-94,208-314$.

[4] Fatou, P.: Sur l'itération des fonctions transcendantes entières. - Acta. Math. 47, 1926, $337-370$.

[5] Golusin, G. M.: Geometrische Funktionentheorie. - VEB Deutscher Verlag der Wissenschaften, Berlin, 1957.

[6] Julia, G.: Mémoire sur l'itération des fonctions rationelles. - J. Math. Pures Appl. (8) 1, 1918, 47-245.

[7] Myrberg, P. J.: Eine Verallgemeinung arithmetisch-geometrischen Mittels. - Ann. Acad. Sci. Fenn. Ser. A I Math. 253, 1958, 1-19.

[8] TsuJ, M.: Potential theory in modern function theory. - Maruzen Co. Ltd., Tokyo, 1959.

Indian Institute of Technology

Department of Mathematics

Madras 600036

India

Received 25 August 1981 